\title{
Photoactivatable mCherry for high-resolution two-color fluorescence microscopy
}

\author{
Fedor V Subach ${ }^{1,3}$, George H Patterson 2,3 , Suliana Manley², Jennifer M Gillette ${ }^{2}$, Jennifer \\ Lippincott-Schwartz ${ }^{2}$, and Vladislav V Verkhusha ${ }^{1}$ \\ ${ }^{1}$ Department of Anatomy and Structural Biology, and Gruss-Lipper Biophotonics Center, Albert \\ Einstein College of Medicine, 1300 Morris Park Ave., Bronx, New York 10461, USA \\ ${ }^{2}$ Section on Organelle Biology, Cell Biology and Metabolism Program, National Institute of Child \\ Health and Human Development, National Institutes of Health, 9000 Rockville Pike, Bethesda, \\ Maryland 20892, USA
}

\begin{abstract}
The reliance of modern microscopy techniques on photoactivatable fluorescent proteins prompted development of mCherry variants that are initially dark but become red fluorescent after violetlight irradiation. Using ensemble and single-molecule characteristics as selection criteria, we developed PAmCherry1 with excitation/emission maxima at 564/595 nm. Compared to other monomeric red photoactivatable proteins, it has faster maturation, better $\mathrm{pH}$ stability, faster photoactivation, higher photoactivation contrast and better photostability. Lack of green fluorescence and single-molecule behavior make monomeric PAmCherry1 a preferred tag for twocolor diffraction-limited photoactivation imaging and for super-resolution techniques such as oneand two-color photoactivated localization microscopy (PALM). We performed PALM imaging using PAmCherry1-tagged transferrin receptor expressed alone or with photoactivatable GFPtagged clathrin light chain. Pair correlation and cluster analyses of the resulting PALM images identified $\leq 200 \mathrm{~nm}$ clusters of transferrin receptor and clathrin light chain at $\leq 25 \mathrm{~nm}$ resolution and confirmed the utility of PAmCherry1 as an intracellular probe.
\end{abstract}

Genetically encoded 'photoactivatable' fluorescent proteins (PAFPs) make up a small category of fluorescent proteins ${ }^{1}$, but are beginning to find uses far and above those of 'normal' fluorescent proteins ${ }^{2}$. With initially little or no fluorescence within their associated spectral detection window, photoactivatable proteins can be switched on by irradiation with violet light. Thus they are useful for spatially pulse-labeling subpopulations of molecules in cells in complement to photobleaching applications and can provide other useful features such as a high contrast over background in the photoactivated region and circumvention of fluorescence contributions from newly synthesized, nonactivated PAFPs. PAFPs and photoswitchable dyes also provide probes necessary for high-resolution optical techniques, such as photoactivated localization microscopy (PALM) ${ }^{3}$, fluorescence photoactivated localization microscopy (FPALM) ${ }^{4}$, stochastic reconstruction microscopy (STORM) ${ }^{5}$, PALM with independent running acquisition (PALMIRA) ${ }^{6}$ and stroboscopic PALM $(\mathrm{SPALM})^{7}$.

(C) 2009 Nature America, Inc. All rights reserved

Correspondence should be addressed to V.V.V. (vverkhus@aecom.yu.edu)..

${ }^{3}$ These authors contributed equally to this work.

AUTHOR CONTRIBUTIONS F.V.S. developed proteins and characterized them in vitro. G.H.P., S.M. and J.M.G. characterized proteins in mammalian cells. J.L.-S. and V.V.V. designed and planned the project. G.H.P. and V.V.V. wrote the manuscript.

Note: Supplementary information is available on the Nature Methods website. 
Of particular interest for PALM is the development of monomeric red PAFPs ${ }^{8}$. Several current variants can be activated into a red-emitting protein, but these have limitations for both live-cell confocal imaging and for high-resolution localization techniques. The obligate tetrameric state of red Kaede ${ }^{9}$, KFP1 (ref. ${ }^{10}$ ) and $\operatorname{EosFP}^{11}$ frequently causes abnormal localization and function of the tagged proteins. Available monomeric red PAFPs such as Dendra $^{12}$, monomeric EosFP (mEosFP) or tandem dimeric EosFP (tdEosFP) ${ }^{11}$ undergo the photoconversion from a green form to a red form, which complicates two-color photoactivation experiments with green $\mathrm{PAGFP}^{13}, \mathrm{PSCFP}^{14}$ or Dronpa ${ }^{15}$. It is also often difficult to achieve in cells a complete green-to-red photoconversion, which results in a detectable residual amount of the green species of these PAFPs. The available PAmRFP1 (ref. 8) switches from a nonfluorescent to a red fluorescent protein, but lacks the photon yields required for PALM applications. Reversibly photoswitchable fluorescent proteins rsCherry and rsCherryRev could be used in two-color experiments with green PAFPs, but have low brightness in their 'on' state, high background fluorescence in their 'off' state and tend to rapidly relax to the dark state after being photoswitched ${ }^{16}$.

Here we report several irreversibly photoactivatable derivatives of mCherry ${ }^{17}$, named PAmCherry proteins, with excellent photoactivation contrast over background, advanced photostability and high single-molecule brightness compatible with PALM imaging.

\section{RESULTS}

\section{Development of photoactivatable mCherry variants}

We analyzed data on color transitions of red fluorescent proteins to the respective nonfluorescent chromoproteins that had been generated by mutagenesis ${ }^{1}$ and identified the corresponding crucial amino acid positions on the basis of the mCherry structure ${ }^{18}$. Positions spatially close to the chromophore, such as 148, 165, 167 and 203 (numbering is in accordance with GFP alignment; Supplementary Fig. 1 online), appear to be major molecular determinants of color ${ }^{10,19}$. We hypothesized that mutagenesis of mCherry at these positions might convert it to a photoactivatable red probe and performed saturating mutagenesis at these positions using the overlap extension approach.

We screened the resulting bacterial library of the site-specific mCherry mutants by fluorescence-activated cell sorting (FACS) and then selected the brightest clones on Petri dishes using a fluorescence stereomicroscope. We identified two weakly photoactivatable mutants with S148L/I165V/Q167P/I203R and S148L/I165L/Q167A/I203R substitutions compared to mCherry. To enhance their characteristics we subjected these mutants to several rounds of random mutagenesis (Supplementary Methods online).

After each round of mutagenesis and sequencing, we purified and analyzed 10-15 candidate mutants in ensemble mode (Supplementary Methods). Then we immobilized 5-10 mutant variants that had the highest product of brightness and photobleaching half-time in ensemble measurements and immobilized them on coverslips for single-molecule imaging using 561 $\mathrm{nm}$ excitation and intermittent low-energy pulses of $405 \mathrm{~nm}$ light. We imaged the molecules until they became photobleached and then analyzed the data as previously described ${ }^{3}$. During the single-molecule screening, we used photon statistics on the localized mCherry mutant proteins as the selection criteria. We recorded the number of molecules localized within the $1 \mathrm{~s}$ integration time for each frame, and the mean and median number of photons for candidate molecules (Supplementary Table 1 online). Finally, we used the mixture of several enhanced variants as a template for the next round of molecular evolution.

After four rounds of random mutagenesis, we selected the three photoactivatable variants exhibiting the best performance in both ensemble and single-molecule modes. As compared 
to parental mCherry, the mutants named PAmCherry1, PAmCherry2 and PAmCherry3 contained E26V/A58T/K69N/L84F/N99K/S148L/I165V/Q167P/L169V/I203R, M18L/ K69N/L84F/A147T/S148L/I165L/Q167A/L169T/A179T/I203R/R226L and M18L/A58T/ K69N/L84F/M137I/E146D/S148L/I165L/Q167A/D178E/I203R substitutions, respectively (Supplementary Fig. 1).

\section{Ensemble characterization of the PAmCherry variants}

Before photoactivation, the purified PAmCherry1, PAmCherry2 and PAmCherry3 had absorbance maxima at 404-406 nm but did not fluoresce when excited at these wavelengths. After photoactivation using a $399 \mathrm{~nm}$ laser line, new absorbance peaks had maxima at 564$570 \mathrm{~nm}$ (Fig. 1a; PAmCherry2 and PAmCherry 3 exhibited similar spectra). In the photoactivated state, PAmCherry1, PAmCherry 2 and PAmCherry3 exhibited red fluorescence with excitation/emission peaks at 564/595 (Fig. 1a), 570/596 and 570/596 nm, respectively. Molar extinction coefficients and quantum yields of the photoactivated PAmCherry proteins were $18,000-24,000 \mathrm{M}^{-1} \mathrm{~cm}^{-1}$ and $0.24-0.53$, respectively (Table 1). After photoactivation, the red forms of the PAmCherry variants were stable with time and did not relax back to the dark state at least for $24 \mathrm{~h}$ at $37{ }^{\circ} \mathrm{C}$ or two weeks at $4{ }^{\circ} \mathrm{C}$. The protein maturation half-times for the PAmCherry variants were 1.2-1.7-fold of that for mCherry, $18-25$ min at $37^{\circ} \mathrm{C}$ (Fig. 1b). In addition, the PAmCherry variants exhibited monomeric behavior similar to that of mCherry when separated on a native polyacrylamide gel (data not shown).

We also compared biochemical and photochemical ensemble characteristics of PAmCherry variants with those of tdEosFP ${ }^{11}$. For ensemble experiments we selected tdEosFP over mEosFP, which we used for the initial single-molecule screening, because the very low amounts of the latter produced even at low temperatures were insufficient for ensemble measurements. The red fluorescence of the PAmCherry variants exhibited higher $\mathrm{pH}$ stability than tdEosFP with apparent $\mathrm{p} K_{\mathrm{a}}$ values of 6.2-6.3 (Fig. 1c). Both the efficiency and rate of photoactivation of PAmCherry1 (Fig. 1d for PAmCherry1; PAmCherry2 and PAmCherry3 exhibited similar dependences), and the other two PAmCherry variants (data not shown) decreased at acidic $\mathrm{pH}$, suggesting that the PAmCherry photoactivation involves a pH-dependent Glu222 decarboxylation. We also concluded that photoactivation of PAmCherry variants was still possible at $\mathrm{pH} 5.5$, albeit at $\sim 10 \%$ of the photoactivation contrast at $\mathrm{pH} 7.2$, which makes them useful for monitoring lysosomes, the most acidic cellular compartments. Measurements of the photoactivation kinetics by epifluorescence microscopy indicated that the purified PAmCherry 1 became photoactivated more than twofold faster than the other two PAmCherry variants and 1.6-fold faster than tdEosFP (Fig. 1e). Under the same conditions, the photoactivated PAmCherry variants photobleached 1.83.5-fold slower than tdEosFP (Fig. 1f). The photoactivation contrast achieved with the purified PAmCherry variants was 3,000-5,000-fold, whereas the increase in the red fluorescence of the purified tdEosFP was 200-fold (Table 1).

As the recently reported mCherry mutants, rsCherry and rsCherryRev, exhibit reversible photoswitching, we checked this property in PAmCherry1 using the irradiation conditions used in the original paper ${ }^{16}$ but expanded the tests to four different irradiation wavelengths in the range of 370-585 nm (Fig. 1). When cyclically irradiated using 390/40 and 570/30 filter combinations, subpopulations of the PAmCherry1 sample were sequentially photoactivated with violet light and then photobleached with yellow light (Fig. 1g). As expected for irreversibly photoactivatable proteins, the majority of PAmCherry1 was photobleached after about 15 light cycles. We did not observe any reversible photoswitching. We observed similar behavior when the 436/20 filter was used instead of the 390/40 filter (Fig. 1h). PAmCherry1 did not exhibit photoactivation when irradiated with blue light through a $480 / 40$ filter (Fig. 1i). The rsCherry and rsCherryRev variants were 
cyclically photoswitched using the same conditions (Fig. 1g-i). Note that for data shown in Figure $1 \mathrm{~g}-\mathrm{i}$, the fluorescence signal of PAmCherry 1 was normalized to its maximal fluorescence achievable when activated using a 390/40 filter in the absence of the photobleaching yellow light.

\section{Evaluation of PAmCherry variants in cells}

We performed confocal microscopy photoactivation experiments in COS-7 cells using untagged PAmCherry variants co-expressed with enhanced GFP (EGFP) as a transfected cell marker (Supplementary Fig. 2a-c online). Initially the cells displayed little fluorescence in the red channel and after photoactivation showed increases of $\sim 100$-fold for all PAmCherry variants in the cytoplasm (Supplementary Fig. 3a online). Normalization to the initial signal indicated a lower photoactivation contrast than that obtained for the purified proteins because cellular autofluorescence increased the initial signal used for normalization.

To further study PAmCherry1 in cells, we fused it to five different proteins, expressed the fusions in cells and imaged them by confocal microscopy before and after photoactivation. All five, including histone $\mathrm{H} 2 \mathrm{~B}, \beta$-actin and $\alpha$-tubulin fusion proteins, properly localized when visualized after photoactivation in live cells (Supplementary Fig. 4 online). For PALM assessments, we fused PAmCherry variants to two plasma membrane proteins, transferrin receptor (TfR) ${ }^{20}$ and a temperature-sensitive version of vesicular stomatitis virus $\mathrm{G}$ protein $(\mathrm{VSVG})^{21}$, which can be readily imaged using total internal reflection (TIRF) microscopy. The TfR has an intracellular pool in early and recycling endosomes that exchange with the plasma membrane population ${ }^{22}$. TfR binds iron-loaded transferrin for iron uptake, and its cellular distribution, endocytic behavior and diffusion characteristics are well-studied. VSVG is often used in secretory pathway studies, and it is a highly expressed plasma membrane protein when cells are maintained at $32{ }^{\circ} \mathrm{C}$.

When expressed in COS-7 cells, TfR-PAmCherry variants (Supplementary Fig. 2d-f) had intracellular pools and plasma membrane populations similar to those of TfR-EGFP, TfRmCherry (Supplementary Fig. 2g) and endogenous TfR as previously reported ${ }^{23}$.

Measurement of transferrin uptake indicated that the chimeras are functional and capable of binding and internalizing transferrin (data not shown). We performed photoactivation kinetics measurements using the more highly expressed VSVG-PAmCherry 1 fusion and observed an $\sim 100$-fold increase in fluorescence at the plasma membrane (Supplementary Fig. 3b). Similar to the results with purified proteins (Fig. 1e), PAmCherry1 in cells activated with less irradiation when compared to the other variants (Supplementary Fig. 3a), which is an important property for live-cell photoactivation experiments.

\section{Evaluation of PAmCherry1 as a PALM marker in cells}

Initial PALM experiments with TfR-PAmCherry variants in COS-7 cells allowed singlemolecule characterization of the PAmCherry variants. The number of photons collected from single molecules revealed that these new variants should work well in cellular imaging experiments (Supplementary Table 1). The slight difference in detected photon yields between cells and purified protein is not surprising, as the proteins are subject to different environments by being attached to TfR and fixed before imaging. In subsequent studies we used PAmCherry1 because of its brightness compared to PAmCherry3, its photostability compared with PAmCherry2 and its easier photoactivation compared to both other proteins (Table 1 and Supplementary Fig. 3).

We directly compared PAmCherry1 (Fig. 2) with rsCherry and rsCherryRev, as well as with tdEosFP, which has been used and characterized in previous PALM studies $3,24,25$. The fluorescence from all frames collected in a PALM experiment with TfR-PAmCherry 1 
produced the equivalent of a diffraction-limited TIRF microscopy image (Fig. 2a).

Comparison of the TIRF microscopy image with the PALM image showed the greater detail in the more highly resolved $(<25 \mathrm{~nm}$ molecular organization uncertainty $(\sigma))$ PALM image (Fig. 2b). A high-magnification TIRF microscopy image showed many bright and dim spots, which corresponded in the PALM image to high or low densities of molecules (Fig. 2d). We performed cluster analysis ${ }^{25}$ to highlight the dense structures, which revealed that many of these heterogeneities correspond to $\sim 100-200 \mathrm{~nm}$ clusters of TfR-PAmCherry1 (Fig. 2c,f).

We performed PALM experiments with TfR-rsCherry- and TfR-rsCherryRev-expressing cells using a modified protocol. The high background from both fluorescent proteins required preirradiations with $488 \mathrm{~nm}$ for rsCherry and $561 \mathrm{~nm}$ for rsCherryRev to switch them off from the ground states. The residual off-state fluorescence for both proteins still presented a problem for single-molecule localization and required an increase in the preactivation irradiation. In addition, experiments with rsCherry were complicated by the reversal of its light sensitivity (Fig. 1g-i). Comparison of the TfR-PAmCherry1 with TfRrsCherry and TfR-rsCherryRev molecules showed that PAmCherry1 had substantially better key PALM properties, such as mean number of photons, mean $\sigma$ values and duration of the molecular fluorescence, than rsCherryRev and rsCherry (Supplementary Fig. 5 online). Notably, the density of molecules in the TfR-rsCherry and TfR-rsCherryRev PALM images was substantially lower than that for TfR-PAmCherry1. This observation may explain why the rsCherry and rsCherryRev produce moderate numbers of photons from single molecules (Supplementary Fig. 5) but have very low brightness in the ensemble (Table 1). The simplest explanation is that there is a large subpopulation of the rsCherry and rsCherryRev molecules, which can form the chromophore, but remain nonfluorescent, that is, in a chromo state $^{1}$, and cannot be photoactivated.

We also compared PAmCherry1 with a more thoroughly characterized PALM probe, tdEosFP ${ }^{3,24,25}$. TfR-tdEosFP did not show the normal punctate intracellular and plasma membrane TfR distributions but was located in the cytosol with several large punctate signals throughout the cytoplasm (Supplementary Fig. 6 online). Nevertheless, sufficient TfR-tdEosFP molecules were within the TIRF microscopy excitation range for comparing properties important for PALM with those of TfR-PAmCherry1 (Fig. 3). In cells imaged and analyzed under the same conditions the number of photons per molecule had slightly different distributions (Fig. 3a) with $\sim 940$ and $\sim 724$ mean number of photons collected for tdEosFP and PAmCherry1, respectively. Despite the slight shift toward more photons per molecule in the distribution of PAmCherry1, tdEosFP has the higher mean due to a population of molecules that produce up to 30,000 photons. $\sigma$ distributions were centered around $\sim 15-16 \mathrm{~nm}$ uncertainty (Fig. 3b) indicating that PAmCherry 1 and tdEosFP were comparable in fixed cell PALM experiments. Analysis of the duration of fluorescence from each molecule revealed that the tdEosFP molecules tend to fluoresce $\sim 1$ frame $(0.1 \mathrm{~s})$ longer than PAmCherry1 (Fig. 3c). Lastly, contrast comparisons made by observation and background quantification of frame 1,000 from 10,000-frame experiments ${ }^{24}$ indicated no substantial difference between PAmCherry1 and tdEosFP in this property. We also compared PAmCherry 1 and tdEosFP tagged to VSVG in PALM experiments. These data were similar to the TfR data and showed that VSVG-PAmCherry 1 produced fewer photons but had a distribution of molecular localization uncertainties similar to that of VSVGtdEosFP (Fig. 3d-f).

\section{Evaluation of PAmCherry1 as a marker in two-color PALM}

TfR internalizes via clathrin-coated pits $^{26}$, which are reported ${ }^{27}$ to have a similar size to the clusters of TfR we observed in images shown in Figure 2. To test the colocalization of TfR and clathrin-coated pits, we made a PAGFP chimera with clathrin light chain (CLC), which localizes to clathrin-coated pits at the cytoplasmic face of the Golgi apparatus and the 
plasma membrane ${ }^{28}$. We observed TfR-PAmCherry1 and PAGFP-CLC clusters in the TIRF microscopy image similar to those observed in the one-color experiments, with molecules located in clustered areas throughout the plasma membrane (Figs. 3 and $4 \mathrm{a}-\mathrm{f}$ ). In addition, TfRPAmCherry 1 was enriched in larger structures extending several micrometers (Fig. $4 a, b)$, which may represent folds in the plasma membrane.

We observed colocalization of TfR-PAmCherry 1 with PAGFP-CLC in the TfRPAmCherry1 TIRF microscopy image clusters as well as in PALM images (Fig. 4g-i), but clusters of TfR and CLC also appeared either completely separate or in close proximity to each other with little overlap. Despite the lack of complete colocalization, pair correlation analysis confirmed similar molecular distributions and a characteristic cluster size of $~ 200$ $\mathrm{nm}$, with more clustering of CLC, as evidenced by a higher $y$-axis intercept (Fig. 4j). Cluster analysis ${ }^{25}$ identified many structures enriched in TfR-PAmCherry 1 and PAGFP-CLC present in at least three different relative distributions (Fig. 4k,1). The shape and size of clusters observed here were often similar to those of clathrin-coated pits observed by electron microscopy ${ }^{27}$ and the different TfR and CLC clusters may represent stages of previously observed and described clathrin-coated pit dynamics ${ }^{28}$. PAGFP-CLC clusters lacking TfR-PAmCherry 1 cargo may correspond to more mature clathrin-coated pits, whereas the TfR-PAmCherry1 clusters without PAGFP-CLC may represent mature uncoated vesicles close to the plasma membrane; clusters in which the two proteins colocalize may represent young pits that have captured the TfR-PAmCherry1 cargo.

\section{DISCUSSION}

Imaging multiple fluorophores in the same specimen is an important improvement of highresolution molecular localization techniques. Two-color high-resolution localization experiments have been previously reported in which the green-to-red photoconvertible protein tdEosFP was paired with the photoswitchable fluorescent protein Dronpa ${ }^{24,29}$, or in which the photoswitchable fluorescent protein rsFastlime was paired with Cy5 in PALMIRA $^{30}$. These studies produced images at $\sim 25-75 \mathrm{~nm}$ resolution on organelles, cytoskeletal elements and extracellular adhesion proteins. However, with exception of PSCFP2 paired with tdEosFP24, photoswitching of one or both fluorophores between light and dark states is a necessary component for these two-color experiments, which may complicate the final results. If a photoactivated molecule switches off, it can be repeatedly switched on and can then be localized again, skewing the molecular distributions in the final high-resolution image. The use of rsCherry proteins ${ }^{16}$ can also result in skewed distributions. As it is not reliant on photoswitching behavior to maintain a low density of molecular signals in two-color PALM studies, PAmCherry1 is a PAFP that can be used to avoid any potential complication associated with repeated localization of the same molecule.

We developed PAmCherry1 in one of the first mutagenesis screens using single-molecule PALM characteristics in addition to ensemble photoactivation experiments as the selection criteria. PAmCherry1 lacks the capability for ratiometric imaging provided by the green-tored photoconvertible fluorescent proteins. It performed substantially better than rsCherry and rsCherryRev in PALM, was similar to tdEosFP in most PALM comparisons, and did not hamper the trafficking and function of the TfR. The absence of a green emission state allows greater flexibility in one-color PALM experiments using a diffraction-limited green fluorescent molecule, in two-color PALM experiments using a dark-to-green PAFP and notably, will enable two-color live-cell and single-particle PALM. Compared to tdEosFP, the smaller monomeric PAmCherry 1 is potentially less disruptive to tagged fusion partners. PAmCherry1 also has higher $\mathrm{pH}$ stability, displays better photostability, produces higher photoactivation contrast and exhibits faster chromophore formation at $37^{\circ} \mathrm{C}$. Thus, PAmCherry 1 simultaneously addresses many disadvantages exhibited by other red PAFPs 
and, as shown here, meets the demands for use in both diffraction-limited microscopy and super-resolution PALM.

\section{METHODS}

\section{Confocal and PALM imaging}

Cell imaging was performed on either a Zeiss LSM510 or on a Leica SP2 laser scanning confocal microscope as described previously ${ }^{3,13}$. Single-molecule imaging was performed as previously described ${ }^{24,25}$ on an Olympus IX81 microscope using an $\times 601.45$ numerical aperture (NA) PlanApoN TIRF objective (Olympus) with the exception that a Dual-View imaging system (Photometrics) was used in the two-color PALM imaging.

\section{Additional methods}

Descriptions of cloning, characterization of proteins in vitro, construction of plasmids, cell cultures and additional details on imaging are available in Supplementary Methods.

\section{Supplementary Material}

Refer to Web version on PubMed Central for supplementary material.

\section{Acknowledgments}

We thank R. Tsien (University of California at San Diego) for providing the pRSETB-mCherry plasmid, J. Wiedenmann (University of Ulm) for providing plasmids encoding EosFP variants, J. Zhang for assistance with flow cytometry, O. Subach for assistance with cell culture and imaging, and E. Betzig and H. Hess for assistance with PALM experiments, analysis and discussion. This work was supported by grants GM070358 and GM073913 from the US National Institutes of Health to V.V.V.

\section{References}

1. Lukyanov KA, Chudakov DM, Lukyanov S, Verkhusha VV. Innovation: Photoactivatable fluorescent proteins. Nat. Rev. Mol. Cell Biol 2005;6:885-891. [PubMed: 16167053]

2. Hell SW. Far-field optical nanoscopy. Science 2007;316:1153-1158. [PubMed: 17525330]

3. Betzig E, et al. Imaging intracellular fluorescent proteins at nanometer resolution. Science 2006;313:1642-1645. [PubMed: 16902090]

4. Hess ST, Girirajan TP, Mason MD. Ultra-high resolution imaging by fluorescence photoactivation localization microscopy. Biophys. J 2006;91:4258-4272. [PubMed: 16980368]

5. Rust MJ, Bates M, Zhuang X. Sub-diffraction-limit imaging by stochastic optical reconstruction microscopy (STORM). Nat. Methods 2006;3:793-795. [PubMed: 16896339]

6. Egner A, et al. Fluorescence nanoscopy in whole cells by asynchronous localization of photoswitching emitters. Biophys. J 2007;93:3285-3290. [PubMed: 17660318]

7. Flors $\mathrm{C}$, et al. A stroboscopic approach for fast photoactivation-localization microscopy with Dronpa mutants. J. Am. Chem. Soc 2007;129:13970-13977. [PubMed: 17956094]

8. Verkhusha VV, Sorkin A. Conversion of the monomeric red fluorescent protein into a photoactivatable probe. Chem. Biol 2005;12:279-285. [PubMed: 15797211]

9. Ando R, Hama H, Yamamoto-Hino M, Mizuno H, Miyawaki A. An optical marker based on the UV-induced green-to-red photoconversion of a fluorescent protein. Proc. Natl. Acad. Sci. USA 2002;99:12651-12656. [PubMed: 12271129]

10. Chudakov DM, et al. Kindling fluorescent proteins for precise in vivo photolabeling. Nat. Biotechnol 2003;21:191-194. [PubMed: 12524551]

11. Wiedenmann J, et al. EosFP, a fluorescent marker protein with UV-inducible green-to-red fluorescence conversion. Proc. Natl. Acad. Sci. USA 2004;101:15905-15910. [PubMed: 15505211] 
12. Gurskaya NG, et al. Engineering of a monomeric green-to-red photoactivatable fluorescent protein induced by blue light. Nat. Biotechnol 2006;24:461-465. [PubMed: 16550175]

13. Patterson GH, Lippincott-Schwartz J. A photoactivatable GFP for selective photolabeling of proteins and cells. Science 2002;297:1873-1877. [PubMed: 12228718]

14. Chudakov DM, et al. Photoswitchable cyan fluorescent protein for protein tracking. Nat. Biotechnol 2004;22:1435-1439. [PubMed: 15502815]

15. Ando R, Mizuno H, Miyawaki A. Regulated fast nucleocytoplasmic shuttling observed by reversible protein highlighting. Science 2004;306:1370-1373. [PubMed: 15550670]

16. Stiel AC, et al. Generation of monomeric reversibly switchable red fluorescent proteins for farfield fluorescence nanoscopy. Biophys. J 2008;95:2989-2997. [PubMed: 18658221]

17. Shaner NC, et al. Improved monomeric red, orange and yellow fluorescent proteins derived from Discosoma sp. red fluorescent protein. Nat. Biotechnol 2004;22:1567-1572. [PubMed: 15558047]

18. Shu X, Shaner NC, Yarbrough CA, Tsien RY, Remington SJ. Novel chromophores and buried charges control color in mFruits. Biochemistry 2006;45:9639-9647. [PubMed: 16893165]

19. Verkhusha VV, Lukyanov KA. The molecular properties and applications of Anthozoa fluorescent proteins and chromoproteins. Nat. Biotechnol 2004;22:289-296. [PubMed: 14990950]

20. Huebers HA, Huebers E, Josephson B, Csiba E. A highly efficient chemical isolation procedure for the rat placental transferrin receptor. Biochim. Biophys. Acta 1989;991:30-35. [PubMed: 2713419]

21. Gallione CJ, Rose JK. A single amino acid substitution in a hydrophobic domain causes temperature sensitive cell-surface transport of a mutant viral glycoprotein. J. Virol 1985;54:374382. [PubMed: 2985803]

22. McGraw TE, Maxfield FR. Human transferrin receptor internalization is partially dependent upon an aromatic amino acid on the cytoplasmic domain. Cell Regul 1990;1:369-377. [PubMed: 2100204]

23. Hopkins CR. The appearance and internalization of transferrin receptors at the margins of spreading human tumor cells. Cell 1985;40:199-208. [PubMed: 2981629]

24. Shroff H, et al. Dual-color super-resolution imaging of genetically expressed probes within individual adhesion complexes. Proc. Natl. Acad. Sci. USA 2007;104:20308-20313. [PubMed: 18077327]

25. Manley S, et al. High-density mapping of single-molecule trajectories with photoactivated localization microscopy. Nat. Methods 2008;5:155-157. [PubMed: 18193054]

26. Pearse BMF, Robinson MS. Clathrin, adaptors, and sorting. Annu. Rev. Cell Biol 1990;6:151-171. [PubMed: 2177341]

27. Heuser JE, Anderson RG. Hypertonic media inhibit receptor-mediated endocytosis by blocking clathrin-coated pit formation. J. Cell Biol 1989;108:389-400. [PubMed: 2563728]

28. Kirchhausen T. Clathrin. Annu. Rev. Biochem 2000;69:699-727. [PubMed: 10966473]

29. Habuchi S, et al. Reversible single-molecule photoswitching in the GFP-like fluorescent protein Dronpa. Proc. Natl. Acad. Sci. USA 2005;102:9511-9516. [PubMed: 15972810]

30. Bock H, et al. Two-color far-field fluorescence nanoscopy based on photoswitchable emitters. Appl. Phys. B 2007;88:161-165. 

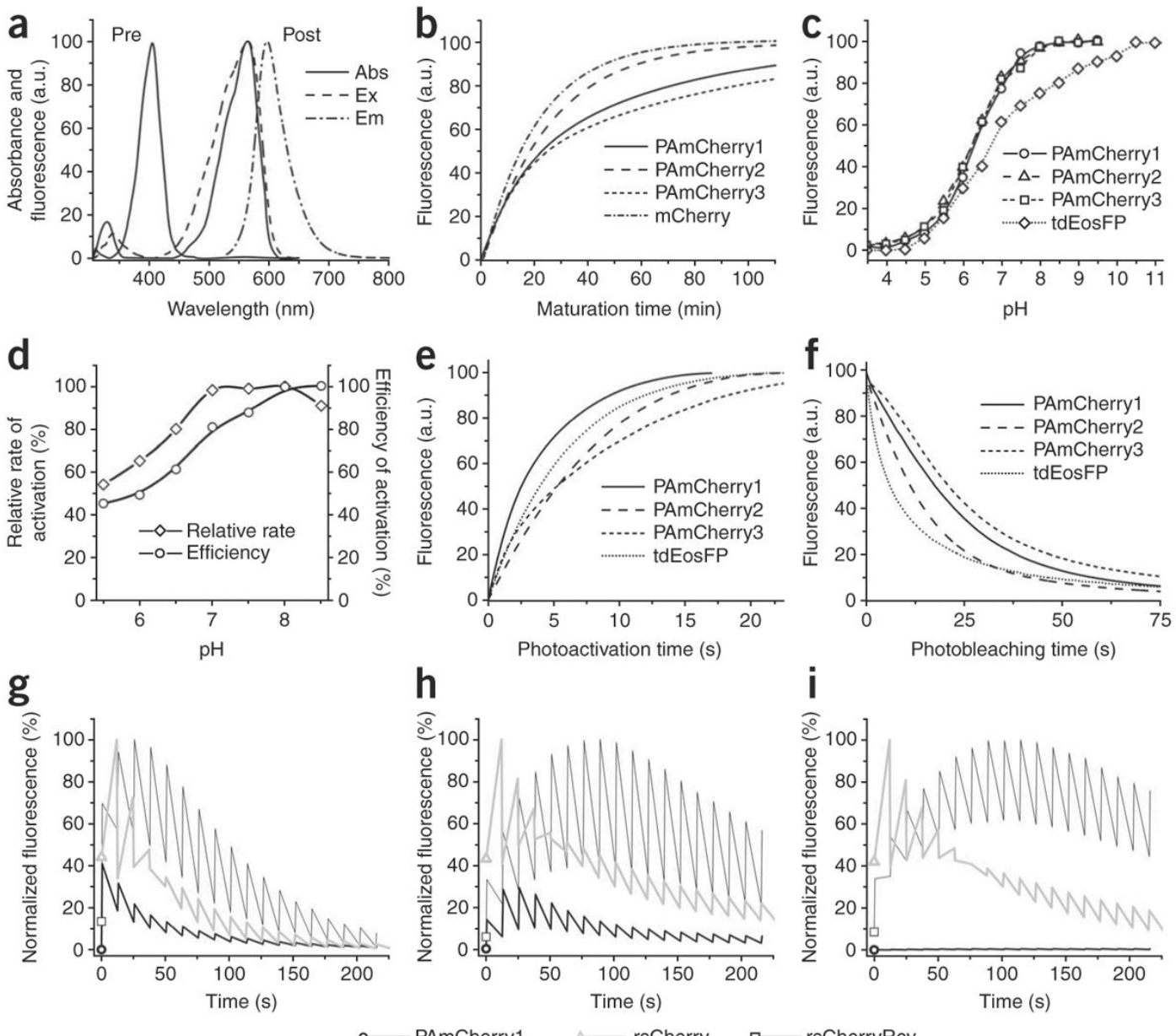

Figure 1.

Spectral, biochemical and photochemical properties of the purified PAmCherry variants. (a) Absorbance, excitation and emission spectra of PAmCherry1 before (pre) and after (post) photoactivation with the $399 \mathrm{~nm}$ laser line. (b) Maturation kinetics for the indicated proteins at $37{ }^{\circ} \mathrm{C}$. (c) Equilibrium $\mathrm{pH}$ dependence for the red fluorescence of the indicated proteins.

(d) Rate and efficiency of PAmCherry1 photoactivation at different physiological $\mathrm{pH}$ values, normalized to the fluorescence intensities at the respective $\mathrm{pH}$ (data shown in $\mathbf{c}$ ). (e,f) Photoactivation (e) and photobleaching (f) kinetics for the indicated proteins using arc lamp illumination through $\times 60$ oil objective with $390 / 40 \mathrm{~nm}(\mathbf{e})$ and $570 / 30 \mathrm{~nm}$ (f) filters. (g-i) Cycling illumination of PAmCherry1, rsCherry and rsCherryRev using an arc lamp, $\times 100$ oil objective, $570 / 30$ filter for $12 \mathrm{~s}$ and one of the following filters: $390 / 40 \mathrm{~nm}$ for $0.6 \mathrm{~s}$ (g), 436/20 for $0.5 \mathrm{~s}$ (h) and 480/40 for $0.4 \mathrm{~s} \mathrm{(i).} \mathrm{The} \mathrm{maxima} \mathrm{of} \mathrm{the} \mathrm{fluorescence} \mathrm{signals}$ shown in $\mathbf{g - i}$ were normalized per the relative brightness for each protein indicated in Table 1 to achieve $100 \%$. The ground states of the proteins are indicated with the respective symbols. 


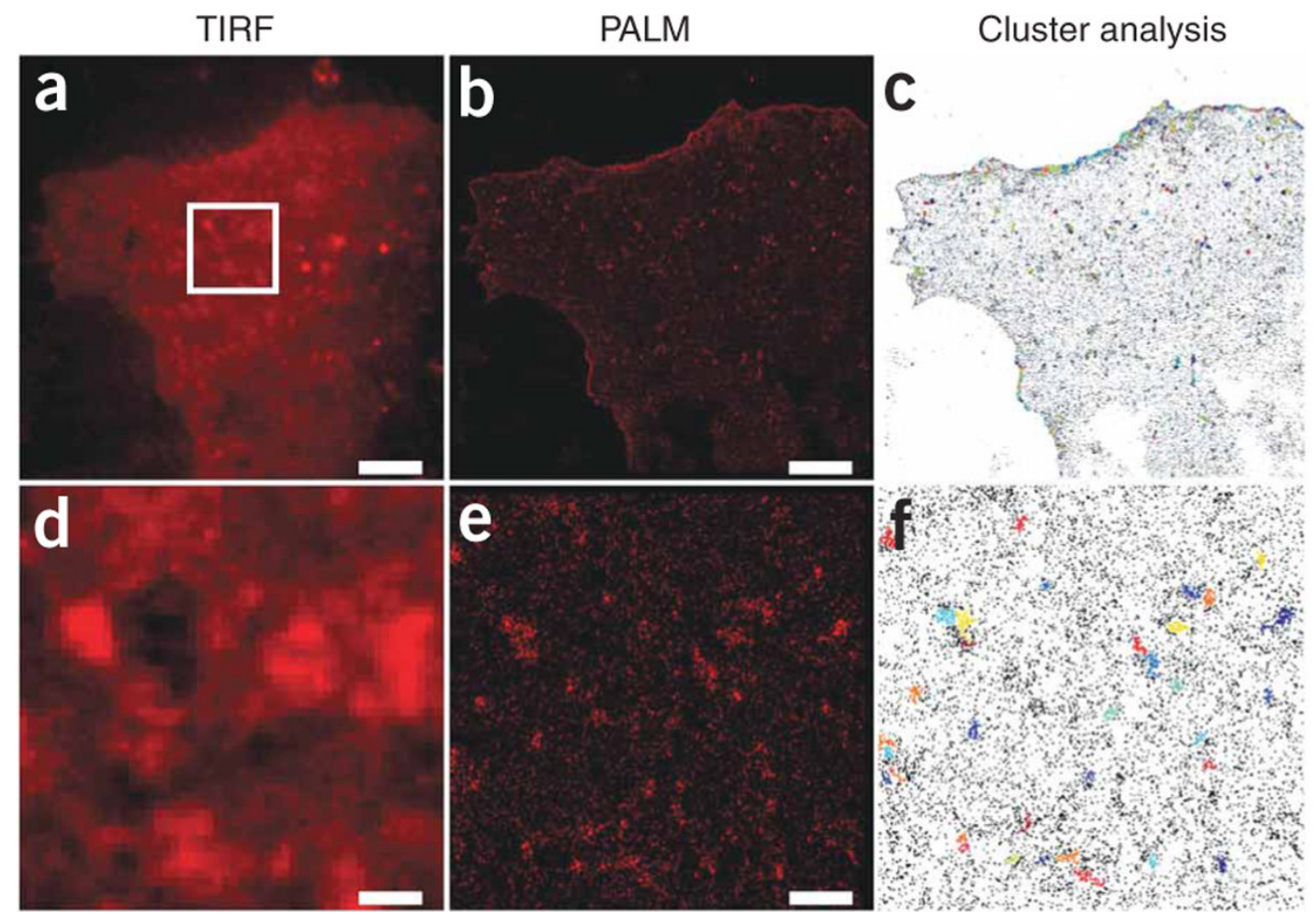

Figure 2.

Plasma membrane distribution of TfR observed using PALM. (a) Cells expressing TfR-

PAmCherry1 were subjected to low-level 405-nm laser photoactivation, and we simultaneously collected 561-nm-laser-light-excited PAmCherry1 single-molecule fluorescence. Data were collected at 10 frames per second for 15,000 frames. The fluorescence collected during the 15,000 frames is shown in a and represents a diffractionlimited TIRF microscopy image. (b) The position and uncertainty of the molecules plotted as Gaussian-normalized spots to form a PALM image. (c) Cluster analysis was performed on the molecules localized over the entire PALM image. The threshold used for clusters required the local molecular density to be fivefold that of the average molecular density. Individual clusters are plotted in different colors with nonclustered molecules represented in black. (d-f) Magnified views of the boxed region in a are shown as TIRF microscopy (d), PALM (e) and cluster analysis images (f). PALM data in these images were limited to molecules that localized to $<25 \mathrm{~nm}$ uncertainty. Scale bars, $5 \mu \mathrm{m}(\mathbf{a , b})$ and $1 \mu(\mathbf{d}, \mathbf{e})$. 

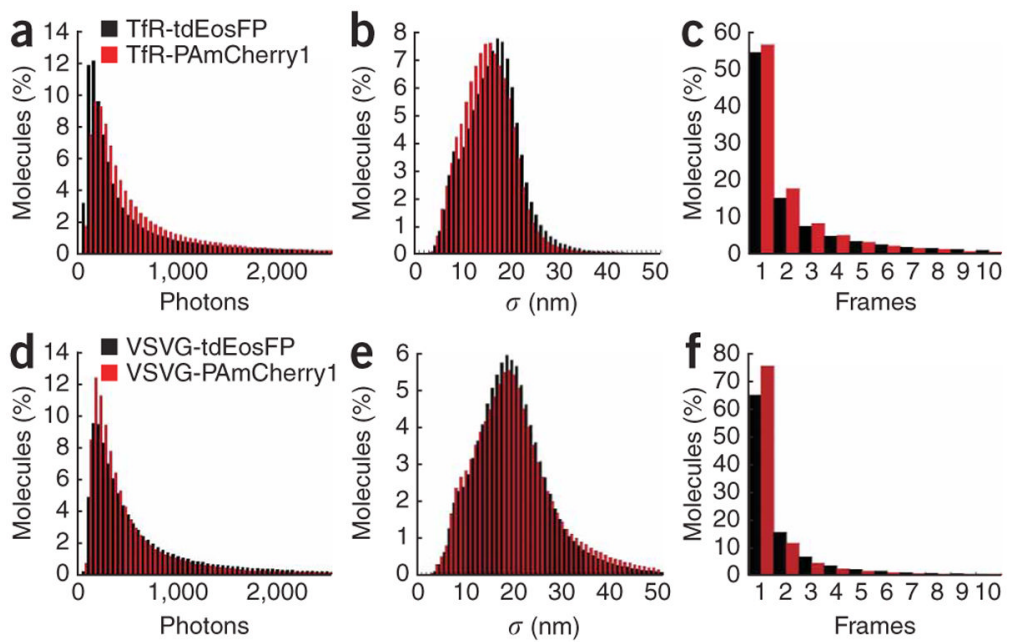

Figure 3.

Comparison of PAmCherry1 and tdEosFP fusions in fixed cells. (a) Distribution of the number of photons collected from molecules in cells expressing TfR-PAmCherry1 (mean, 724; median, 413) and TfR-tdEosFP (mean, 940; median, 349). (b) Distribution of $\sigma$ in cells expressing TfR-PAmCherry1 (mean, 15.18; median, 14.99) and TfR-tdEosFP (mean, 16.75; median, 16.69). (c) Distribution of the duration of the molecular fluorescence (0.1-s frames) in cells expressing TfR-PAmCherry1 (mean, 2.34; median, 1) and TfR-tdEosFP (mean, 3.26; median, 1). Two-tailed Wilcoxon-Mann-Whitney tests indicated differences between the TfR-PAmCherry 1 and TfR-tdEosFP data medians for all three distributions $(P<0.0001$, $n=757,180$ from five TfR-PAmCherry 1 -expressing cells and $n=656,290$ from five TfRtdEosFP-expressing cells). (d) Distribution of the number of photons collected from molecules in cells expressing VSVG-PAmCherry1 (mean, 657; median, 348) and VSVGtdEosFP (mean, 1,057; median, 444). (e) Distribution of $\sigma$ in cells expressing VSVGPAmCherry1 (mean, 20.96; median, 19.68) and VSVG-tdEosFP (mean, 19.91; median, 19.04). (f) Distribution of the duration of the molecular fluorescence (0.1-s frames) in cells expressing VSVG-PAmCherry1 (mean, 1.82; median, 1) and VSVG-tdEosFP (mean, 2.37; median, 1). Two-tailed Wilcoxon-Mann-Whitney tests indicated differences between the VSVG-PAmCherry1 and VSVG-tdEosFP data medians for all three distributions $(P<$ $0.0001, n=377,010$ from three VSVG-PAmCherry 1 cells and $n=768,040$ from three VSVG-tdEosFP cells). 


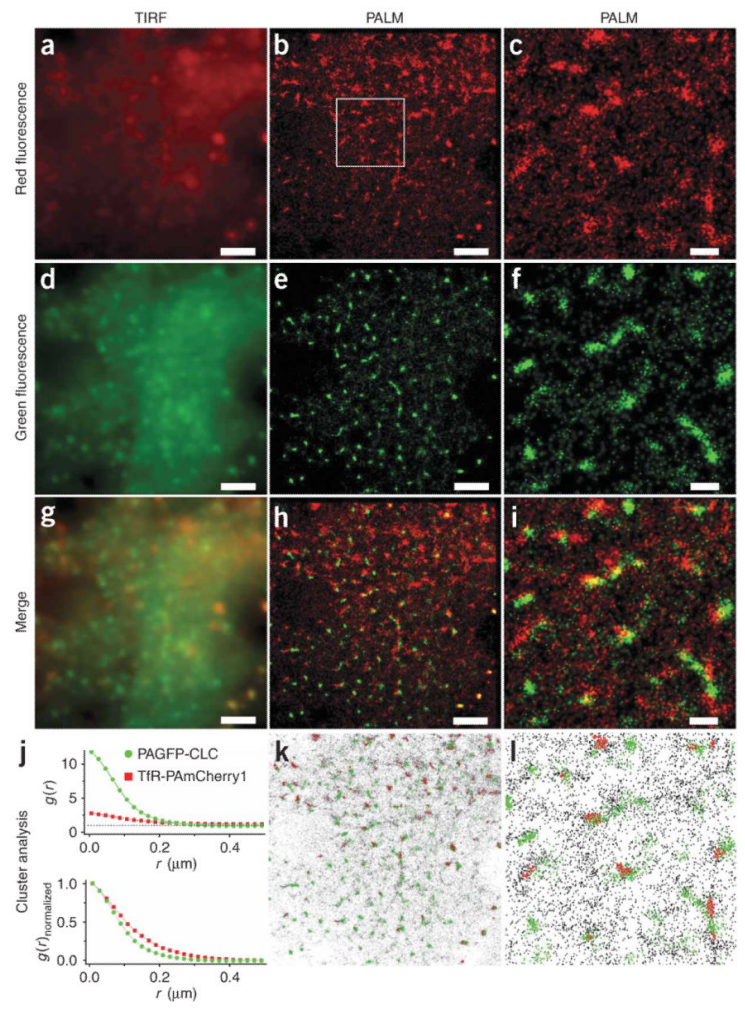

Figure 4.

Distributions of TfR and CLC by two-color PALM. (a-i) COS-7 cells expressing TfR-

PAmCherry1 and PAGFP-CLC were subjected to low-level 405-nm laser photoactivation while we alternately collected 561-nm-light-excited PAmCherry1 (a-c) and 488-nm-lightexcited PAGFP (d-f) single-molecule fluorescence. TIRF microscopy images of

PAmCherry1 fluorescence (a), PAGFP fluorescence (d) and the merge (g) are shown. Position and uncertainty for TfR-PAmCherry $1(\mathbf{b}, \mathbf{c})$ and PAGFP-CLC $(\mathbf{e , f})$ are plotted as Gaussian-normalized spots. Magnified views of the boxed area in $\mathbf{b}$ are shown in $\mathbf{c}, \mathbf{f}$ and $\mathbf{i}$. Merge images (g-i) show the relative distributions of TfR-PAmCherry1 (red) and PAGFPCLC (green). (j) Pair correlation analysis (top) and normalized results (bottom) for the presence and size of TfR-PAmCherry1 and PAGFP-CLC clusters. (k) Cluster analysis of the molecules localized for the PALM images in $\mathrm{h}(\mathbf{k})$ and $\mathbf{i}(\mathbf{l})$. The threshold used for clusters required the local molecular density to be fivefold that of the average molecular density. TfR-PAmCherry1 clusters are plotted in red, PAGFP-CLC clusters in green and nonclustered molecules in black. PALM data in these images are limited to molecules that localized to $<25 \mathrm{~nm} \sigma$. Scale bars, $2 \mu \mathrm{m}(\mathbf{a}, \mathbf{b}, \mathbf{d}, \mathbf{e}, \mathbf{g}, \mathbf{h})$ and $0.5 \mu \mathrm{m}(\mathbf{c}, \mathbf{f}, \mathbf{i})$. 


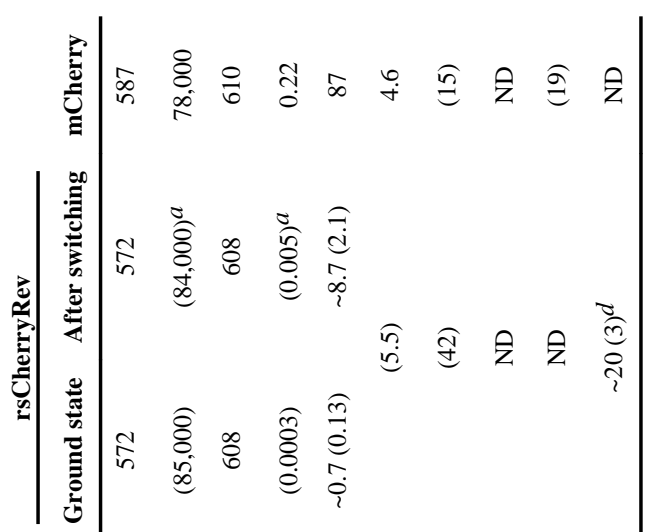

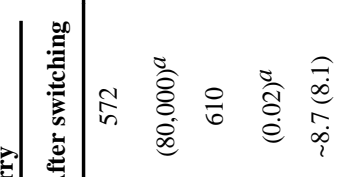
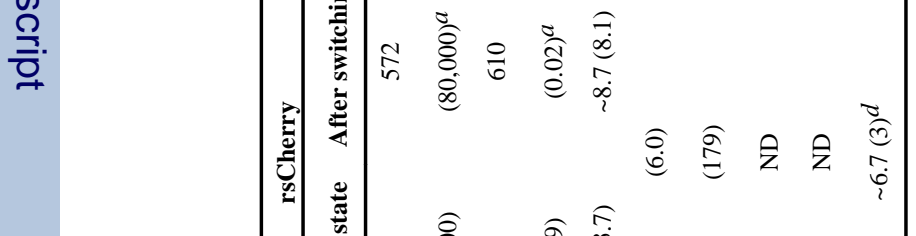

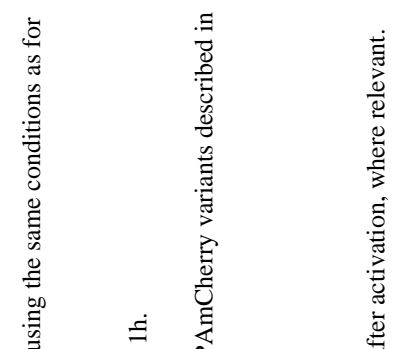

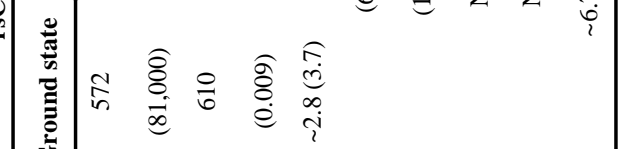

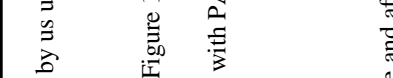

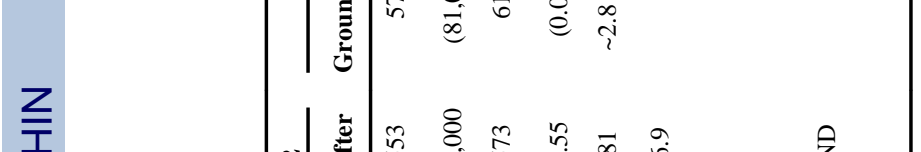

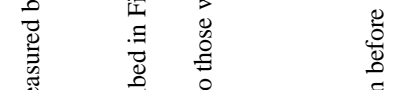

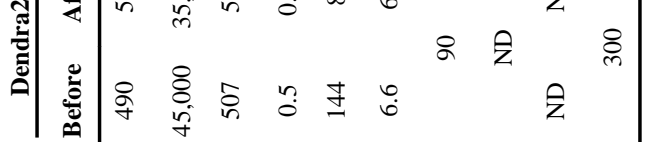
के

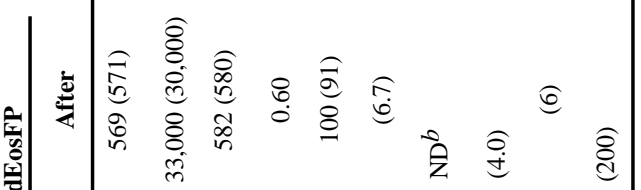

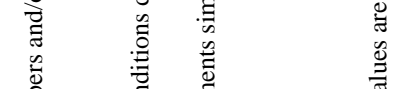

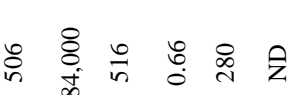

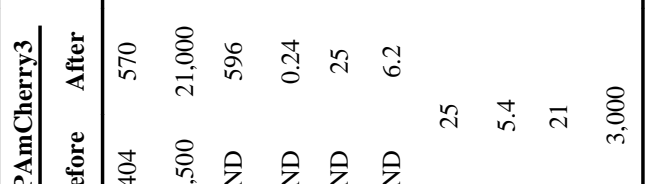

